\title{
Article
}

\section{Visible-Light-Assisted Photoelectrochemical Biosensing of Uric Acid Using Metal-Free Graphene Oxide Nanoribbons}

\author{
Chia-Liang Sun $1,2, * \mathbb{D}$, Cheng-Hsuan Lin ${ }^{1}$, Chia-Heng Kuo ${ }^{1}$, Chia-Wei Huang ${ }^{1}$, Duc Dung Nguyen ${ }^{3}$, \\ Tsu-Chin Chou ${ }^{4}$, Cheng-Ying Chen ${ }^{5}$ and Yu-Jen $\mathrm{Lu}^{2, *}$ \\ 1 Biomedical Engineering Research Center, Department of Chemical and Materials Engineering, \\ Chang Gung University, Taoyuan City 333323, Taiwan; ilovetaihsi@gmail.com (C.-H.L.); \\ w255400@gmail.com (C.-H.K.); jarvis5020huang@gmail.com (C.-W.H.) \\ 2 Department of Neurosurgery, Linkou Chang Gung Memorial Hospital, Taoyuan City 333423, Taiwan \\ 3 Center for High Technology Development, Vietnam Academy of Science and Technology, \\ Hanoi 100000, Vietnam; ddnguyen161@gmail.com \\ 4 Institute of Analytical and Environmental Sciences, National Tsing Hua University, Hsinchu 300044, Taiwan; \\ tsuchin.chou@mx.nthu.edu.tw \\ 5 Center for Plasma and Thin Film Technologies (CPTFT), Department of Materials Engineering, \\ Ming Chi University of Technology, New Taipei City 243303, Taiwan; cychen0111@mail.mcut.edu.tw \\ * Correspondence: sunchialiang@gmail.com or clsun@mail.cgu.edu.tw (C.-L.S.); luyj@cgmh.org.tw (Y.-J.L.)
}

check for updates

Citation: Sun, C.-L.; Lin, C.-H.; Kuo, C.-H.; Huang, C.-W.; Nguyen, D.D.; Chou, T.-C.; Chen, C.-Y.; Lu, Y.-J. Visible-Light-Assisted

Photoelectrochemical Biosensing of Uric Acid Using Metal-Free Graphene Oxide Nanoribbons. Nanomaterials 2021, 11, 2693. https://doi.org/ 10.3390/nano11102693

Academic Editor: Simone Morais

Received: 21 September 2021

Accepted: 10 October 2021

Published: 13 October 2021

Publisher's Note: MDPI stays neutral with regard to jurisdictional claims in published maps and institutional affiliations.

\begin{abstract}
In this study, we demonstrate the visible-light-assisted photoelectrochemical (PEC) biosensing of uric acid (UA) by using graphene oxide nanoribbons (GONRs) as PEC electrode materials. Specifically, GONRs with controlled properties were synthesized by the microwave-assisted exfoliation of multi-walled carbon nanotubes. For the detection of UA, GONRs were adopted to modify either a screen-printed carbon electrode (SPCE) or a glassy carbon electrode (GCE). Cyclic voltammetry analyses indicated that all Faradaic currents of UA oxidation on GONRs with different unzipping/exfoliating levels on SPCE increased by more than $20.0 \%$ under AM 1.5 irradiation. Among these, the GONRs synthesized under a microwave power of $200 \mathrm{~W}$, namely GONR(200 W), exhibited the highest increase in Faradaic current. Notably, the GONR(200 W)/GCE electrodes revealed a remarkable elevation $(40.0 \%)$ of the Faradaic current when irradiated by light-emitting diode (LED) light sources under an intensity of illumination of $80 \mathrm{~mW} / \mathrm{cm}^{2}$. Therefore, it is believed that our GONRs hold great potential for developing a novel platform for PEC biosensing.
\end{abstract}

Keywords: GONR; MWCNT; uric acid; photoelectrochemical; LED

\section{Introduction}

In recent years, nanocarbon materials, including graphene nanoribbons (GNRs), have been of great interest to the scientific community and have been widely investigated for various applications [1-5]. Among different research fields, nanocarbon-based electrochemical biosensors have attracted significant attention due to the tremendous demands of our modern society. Conversely, semiconductor materials or earth-abundant catalysts have been studied for their photoelectrochemical (PEC) activities, such as energy conversion and water splitting, including hydrogen evolution reaction and oxygen evolution reaction [6-8]. Interestingly, semiconductor electrocatalysts with PEC properties can be exploited as photoelectrodes for PEC biosensors [9-20]. For example, Li et al. used $\mathrm{TiO}_{2} / \mathrm{g}$ $\mathrm{C}_{3} \mathrm{~N}_{4}$ in conjunction with dendrimer and alkaline phosphatase for the sensitive detection of protein kinase A activity [9]. Lin et al. combined carbon quantum dots-functionalized $\mathrm{MnO}_{2}$ nanosheets with glucose oxidase $\left(\mathrm{GO}_{\mathrm{x}}\right)$, labeled as $\mathrm{AFB}_{1}$-bovine serum albumin, for monitoring aflatoxin $\mathrm{B}_{1}[10]$. Zhou et al. synthesized reduced graphene oxide $/ \mathrm{BiFeO}_{3}$ nanohybrids on a magnetic microfluidic device for detection of a prostate-specific antigen [12]. More recently, Sun et al. fabricated a CdS- $\operatorname{In}_{2} S_{3}$ heterojunction for detecting bleomycin [13]. Zhu et al. prepared ultrathin PtNi nanozyme and benzene-ring doped 
g- $\mathrm{C}_{3} \mathrm{~N}_{4}$ for the ultrasensitive sensing of chloramphenicol [15]. Detailed information on recently developed PEC biosensors that use a variety of photoactive materials and light sources is summarized in Table $1[9,10,12-20]$.

Table 1. Summary of PEC biosensors that use a variety of materials and light sources.

\begin{tabular}{|c|c|c|c|c|c|}
\hline Material & Electrode & Analyte & Light Source & Method & Ref. \\
\hline $\begin{array}{l}\mathrm{TiO}_{2} / \mathrm{g}-\mathrm{C}_{3} \mathrm{~N}_{4} \mathrm{w} \text { dendrimer, and } \\
\text { alkaline phosphatase }\end{array}$ & ITO & Protein Kinase A & $\mathrm{N} / \mathrm{A}$ & $\mathrm{I}-\mathrm{t}$ & [9] \\
\hline $\begin{array}{l}\mathrm{CQDs}^{a} \text {-functionalized } \mathrm{MnO}_{2} \\
\mathrm{NSs}^{b} \text { with } \mathrm{GO}_{\mathrm{x}} \text {-labeled } \\
\mathrm{AFB}_{1} \text {-bovine serum albumin }\end{array}$ & FTO & Aflatoxin $\mathrm{B}_{1}$ & $\begin{array}{l}500 \mathrm{~W} \text { Xe lamp with a } 420 \mathrm{~nm} \\
\text { cutoff filter }\end{array}$ & $\mathrm{I}-\mathrm{t}$ & [10] \\
\hline $\mathrm{rGO}^{c} / \mathrm{BiFeO}_{3}$ & FTO & Prostate-specific antigen & LED light $(10 \mathrm{~W})$ & I-t & [12] \\
\hline $\mathrm{CdS}-\mathrm{In}_{2} \mathrm{~S}_{3}$ & ITO & Bleomycin & Xe lamp $(\lambda>420 \mathrm{~nm})$ & $\mathrm{I}-\mathrm{t}$ & [13] \\
\hline 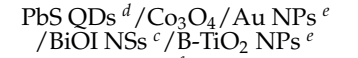 & ITO & Procalcitonin & $\mathrm{N} / \mathrm{A}$ & $\mathrm{I}-\mathrm{t}$ & {$[14]$} \\
\hline $\mathrm{PtNi} \mathrm{NWs}^{f} / \mathrm{BR}^{-\mathrm{CN}}{ }^{g}$ & FTO & Chloramphenicol & $300 \mathrm{~W}$ Xe lamp $(\lambda>420 \mathrm{~nm})$ & I-t & [15] \\
\hline $\begin{array}{c}\text { PDANS }^{h}-\mathrm{CdS}_{\mathrm{QDs}}{ }^{d} / \mathrm{Ag} \\
/ / \mathrm{ERGO}^{i}-\mathrm{TiO}_{2}\end{array}$ & ITO & Prostate specific antigen & $\begin{array}{c}\text { LED } \\
\left(20 \mathrm{~mW} / \mathrm{cm}^{2}\right)\end{array}$ & I-t & [16] \\
\hline $\mathrm{CDs}^{j}-\mathrm{GO}^{k} / \mathrm{Au}-\mathrm{CuO}-\mathrm{Cu}_{2} \mathrm{O}$ & GCE & Progesterone & Xe lamp $(\lambda>420 \mathrm{~nm})$ & I-t & [17] \\
\hline $\mathrm{PDDA}^{l} /$ graphene $/ \mathrm{TiO}_{2}$ & Gold on $\mathrm{SiO}_{2} / \mathrm{Si}$ & Hexavalent chromium & $150 \mathrm{~W}$ Xe lamp & $\mathrm{I}-\mathrm{t}, \mathrm{CV}$ & [18] \\
\hline $\mathrm{Bi}^{3+} / \mathrm{B}^{-} \mathrm{TiO}_{2} / \mathrm{rGO}^{b}$ & ITO & Tobramycin & $250 \mathrm{~W}$ Xe lamp $(\lambda>400 \mathrm{~nm})$ & I-t & [19] \\
\hline $\mathrm{Ag} \mathrm{NCs}{ }^{m} / \mathrm{Ag} \mathrm{NPs}^{e} / \mathrm{GO}$ & Gold & Dopamine and glutathione & $\begin{array}{c}\text { LED (365 nm, } 190 \mathrm{~mW}) \\
\text { LED }\end{array}$ & I-v, CV & [20] \\
\hline $\mathrm{GONR}^{n}$ & GCE & Uric acid & $\begin{array}{c}\text { (6 intensities as well as } \\
6 \text { wavelengths) }\end{array}$ & CV, DPV & This work \\
\hline
\end{tabular}

${ }^{a}$ CQDs: carbon quantum dots. ${ }^{b}$ rGO: reduced graphene oxide. ${ }^{c}$ NSs: nanosheets. ${ }^{d}$ QDs: quantum dots. ${ }^{e}$ NPs: nanoparticles. ${ }^{f}$ NWs: nanowires. ${ }^{g}$ BR-CN: benzene-ring doped g- $\mathrm{C}_{3} \mathrm{~N}_{4} .{ }^{h}$ PDANS: quinone-rich polydopamine nanospheres. ${ }^{i}$ ERGO: electrochemically reduced graphene oxide. ${ }^{j}$ CDs: carbon dots. ${ }^{k}$ GO: graphene oxide. ${ }^{l}$ PDDA: poly(diallyldiamine chloride). ${ }^{m}$ NCs: nanoclusters. ${ }^{n}$ GONR: graphene oxide nanoribbon.

GNRs exhibit extraordinary properties, such as superlubricity, band structure, magnetism, charge/spin transports, high-capacity energy storage, and topological behavior, which are not present in other nanocarbons [21-24]. As for electrochemical biosensors, Vukojević et al. developed $\mathrm{RuO}_{2} / \mathrm{GNR}$-modified screen-printed carbon electrodes to enhance the electrocatalytic biosensing of ethanol and nicotinamide [25]. Tang et al. employed N-GNRs-Fe-MOFs@Au nanocomposites to modify a glassy carbon electrode (GCE) in order to detect Galectin-3, a biomarker for heart failure [26]. Li et al. used nitrogen-doped GNRs and n-C60-PdPt as a dual-type responsive electrochemical immunosensor for the quantitative detection of proprotein convertase subtilisin/kexin type 9 [27]. Feng et al. utilized a combination of gold nanocages and GNRs with large specific surface areas and excellent electrical conductivities to develop a stochastic DNA walker [28]. Liu et al. modified a glassy carbon electrode with a heterostructure of core-shell multi-walled carbon nanotubes at reduced graphene oxide nanoribbons (GONRs) for glutathione detection [29]. Pajooheshpour et al. combined Au-Pt nanoclusters, bovine serum albumin, and GNRs to modify a GCE for the electrochemical detection of diazinon [30]. Although numerous studies have focused on nanocarbon-based biosensors, there is little information available on light sources and their effects on the PEC activities of GONRs. In this context, and based on our previous studies [31-36], we herein report visible-light-assisted PEC biosensors for the detection of uric acid (UA) by using GONRs with controllable properties. The PEC sensing activities of GONRs, synthesized under different microwave powers for the detection of UA, are systematically investigated via a solar simulator. Furthermore, the effects of wavelengths and irradiation intensities of light-emitting diode (LED) light sources on the Faradaic currents of UA oxidation are explored. These findings are especially interesting for the rational design of miniature power-saving devices or systems based on the PEC activities of GONRs towards point-of-care testing.

\section{Experimental Section}

\subsection{Chemicals}

MWCNTs (Ctube 120, CNT Co., Ltd., Songdo, Korea) were purchased from a new international vendor in replacement of an old vendor in our earlier publications [31-36]. The reason being that the original Mistui company has closed their production line in 
recent years [37]. All the other reagents were of analytical grade and used without further purification.

\subsection{Preparation of GONRs}

The GONRs and their electrodes were prepared by a focused microwave reactor (CEM-Discover, CEM, Charlotte, NC, USA) and a freeze dryer (FDS-1000, EYELA, Tokyo, Japan), following the similar procedures in our previous papers [31-36]. It is worthwhile to mention that the nanoribbon powders were collected and placed for drying in a freeze dryer overnight. The ribbon powders were mixed with water, ethanol, and Nafion to form a suspension. $10 \mu \mathrm{L}$ of the suspension was dropped onto either a screen-printed carbon electrode or a glassy carbon electrode, followed by vacuum drying, to prepare a working electrode.

\subsection{Characterization}

The TEM (JEM-1230, JEOL, Tokyo, Japan) studies were performed to monitor the morphology evolution at an operating voltage of $100 \mathrm{kV}$. X-ray photoelectron spectroscopy (XPS) (ESCALAB 250, VG Scientific, Waltham, MA, USA) was used to analyze the changes in composition and bonding. The Raman spectra were characterized by a Raman spectrometer (UniDRON, UniNanoTech, Yongin, Korea), with a laser of $532 \mathrm{~nm}$.

\subsection{Electrochemical and Photoelectrochemical Measurements}

A $3 \mathrm{~mm}$ diameter glassy carbon electrode (002012, ALS Co., Inc., Tokyo, Japan), a $\mathrm{Ag} / \mathrm{AgCl}$ electrode (RE-1S, BAS Inc., Tokyo, Japan), and a platinum wire were used as a working electrode, a reference electrode, and a counter electrode, respectively. Cyclic voltammetry and differential pulsed voltammetry measurements were performed by an electrochemical analyzer (CHI7062E, CH Instruments, Inc., Austin, TX, USA). All solutions were prepared with de-ionized water with a resistivity of $18 \mathrm{M} \Omega / \mathrm{cm}$. Either a solar simulator (Newport, SP94022A, Newport, Irvine, CA, USA) or a wavelength-switchable LED (WLS-22-A, Mightex, T.O., Canada) were provided as the light source. For the PEC measurement, the distance between the light source and the working electrode was normally kept as $10 \mathrm{~cm}$. When we set up the light intensity, the absorption of the wall of a quartz beaker and the electrolyte were ignored. Through a computer, we could adjust the current of an LED to change the light intensity. For example, the current of the blue light bulb of an LED is around $300 \mathrm{~mA}$ for the light intensity of $80 \mathrm{~mW} / \mathrm{cm}^{2}$, as determined by a power meter (StarLite, Ophir, Darmstadt, Germany).

\section{Results and Discussion}

\subsection{Characterization}

Figure 1a displays transmission electron microscopy (TEM) images of the as-purchased / untreated MWCNT, verifying the hollow structure of the material. A microwave-assisted reaction was utilized to unzip the MWCNT in a mixed acid solution. During the reaction, functional groups that contained oxygen could connect to the sidewalls and both ends of the nanotubes, providing attack sites for permanganate ions. Then, sufficient energy input cleaved local carbon-carbon bonds at the outer layers of the nanotubes. Consequently, the nanotubes were gradually unzipped into nanoribbons [31,32]. TEM images of the GONRs unzipped at different microwave powers are shown in Figure $1 \mathrm{~b}-\mathrm{f}$. The longitudinal length of the GONRs was not considerably different from that of the MWCNTs, demonstrating that the reaction did not shorten the nanotubes. Conversely, the widths of the GONRs were wider than the diameter of the MWCNTs. Compared to other ribbons at higher microwave powers, the sidewalls of GONR $(50 \mathrm{~W})$ did not seem to be completely unzipped. Furthermore, the edges of all the ribbon samples were not as continuous as that of our early study, and always contained some broken parts within a certain distance. Figure S1a illustrates the Raman spectra of MWCNTs and GONRs at the excitation laser wavelength of $532 \mathrm{~nm}$. All spectra revealed two main bands, the G band $\left(\sim 1580 \mathrm{~cm}^{-1}\right)$ and the D 
band $\left(\sim 1350 \mathrm{~cm}^{-1}\right)$, indicating the characteristics of nanocarbon materials [38,39]. It was found that the $\mathrm{I}_{\mathrm{D}} / \mathrm{I}_{\mathrm{G}}$ ratios (Figure $\mathrm{S} 1 \mathrm{~b}$ ) of all ribbons were higher than that of MWCNT, indicating the highest value for GONRs unzipped at $50 \mathrm{~W}$. This can be ascribed to the microwave power $(50 \mathrm{~W})$ being insufficient for completely unzipping the MWCNTs yet being high enough for a generation of abundant oxygen-containing groups, consequently contributing to the largest amount of defective sites (i.e., highest $\mathrm{I}_{\mathrm{D}} / \mathrm{I}_{\mathrm{G}}$ ratio). On the contrary, greater powers (100-250 W) could help in unzipping the nanotubes into ribbons at larger extents, thereby possessing more graphitic regions (from the inner walls of the MWCNTs), i.e., contributing more graphitic Raman signals (lower $\mathrm{I}_{\mathrm{D}} / \mathrm{I}_{\mathrm{G}}$ ratios). These results were in good agreement with the TEM observation, confirming that GONR(50 W) was not entirely unzipped.
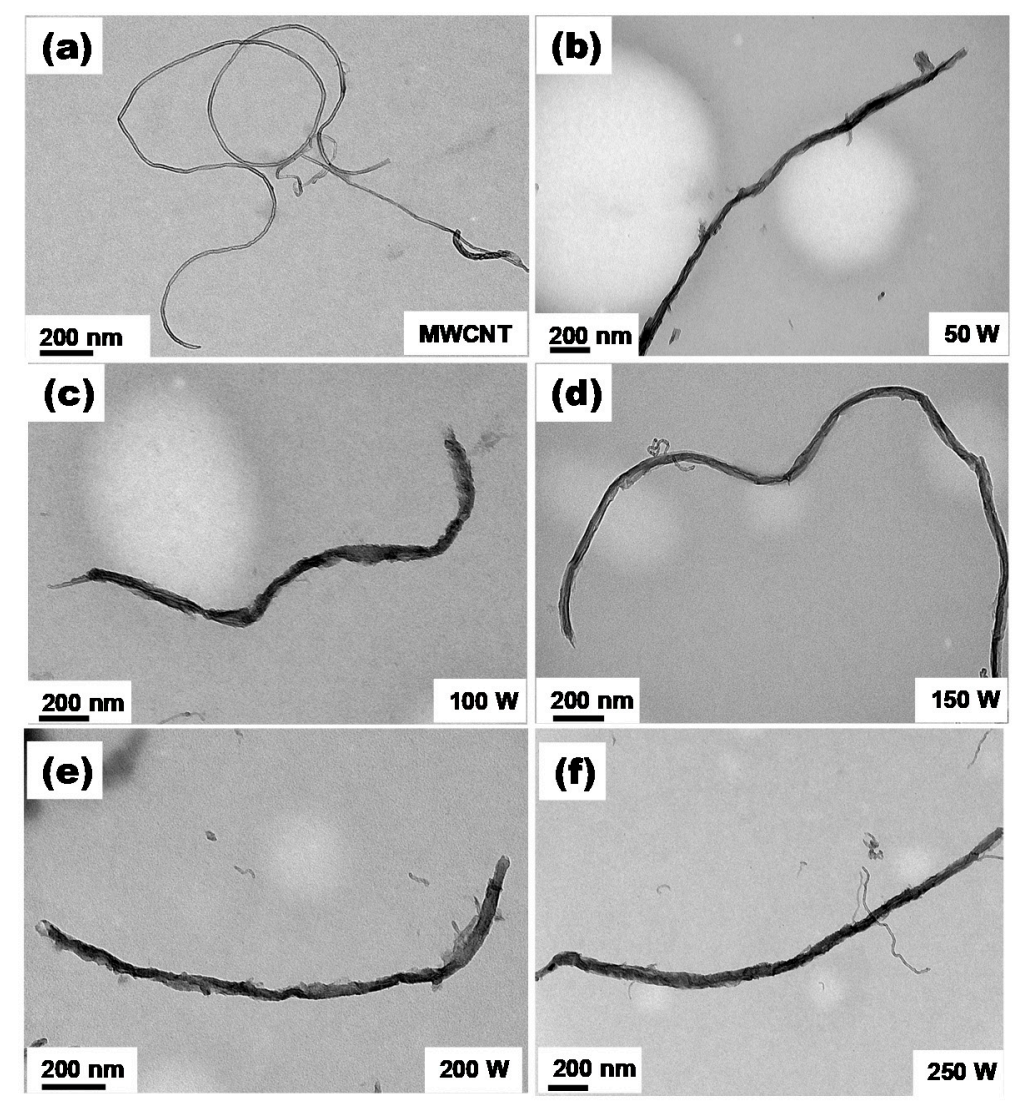

Figure 1. TEM images of (a) MWCNT, (b) GONR(50 W), (c) GONR(100 W), (d) GONR(150 W), (e) GONR(200 W), and (f) GONR(250 W) before and after the microwave-assisted unzipping process at different microwave powers.

X-ray photoelectron spectroscopy (XPS) was adopted to determine the surface compositions and the types of functional groups of the MWCNT and the GONRs. Figure 2 shows the XPS C1s spectra of MWCNT and GONR $(50 \sim 250 \mathrm{~W})$. The deconvolution peaks belonged to $\mathrm{C}-\mathrm{C} / \mathrm{C}=\mathrm{C}(284.5 \mathrm{eV}), \mathrm{C}-\mathrm{OH}(286.0 \mathrm{eV}), \mathrm{C}-\mathrm{O}-\mathrm{C}(287.3 \mathrm{eV}), \mathrm{C}=\mathrm{O}(288.7 \mathrm{eV})$, and $\mathrm{O}-\mathrm{C}=\mathrm{O}(290.4 \mathrm{eV})$. The ratios of different functional groups of the MWCNT and the GONRs, derived from Figure 2, are summarized in Figure S2. In Figure S2, it is noticed that the $\mathrm{C}=\mathrm{O}$ and $\mathrm{O}-\mathrm{C}=\mathrm{O}$ concentrations rose along with increasing microwave powers. Meanwhile, $\mathrm{C}-\mathrm{OH}$ concentration reached the highest value at $50 \mathrm{~W}$ and later declined, implying that the $\mathrm{C}-\mathrm{OH}$ group might have joined the reaction through the unzipping process. Some $\mathrm{C}-\mathrm{OH}$ groups remained on the walls without completely unzipping at higher powers. Besides the surface analysis, elemental analyses confirmed the weight ratios of $\mathrm{C}$ and $\mathrm{O}$ for all samples. The similar trends can be observed. 

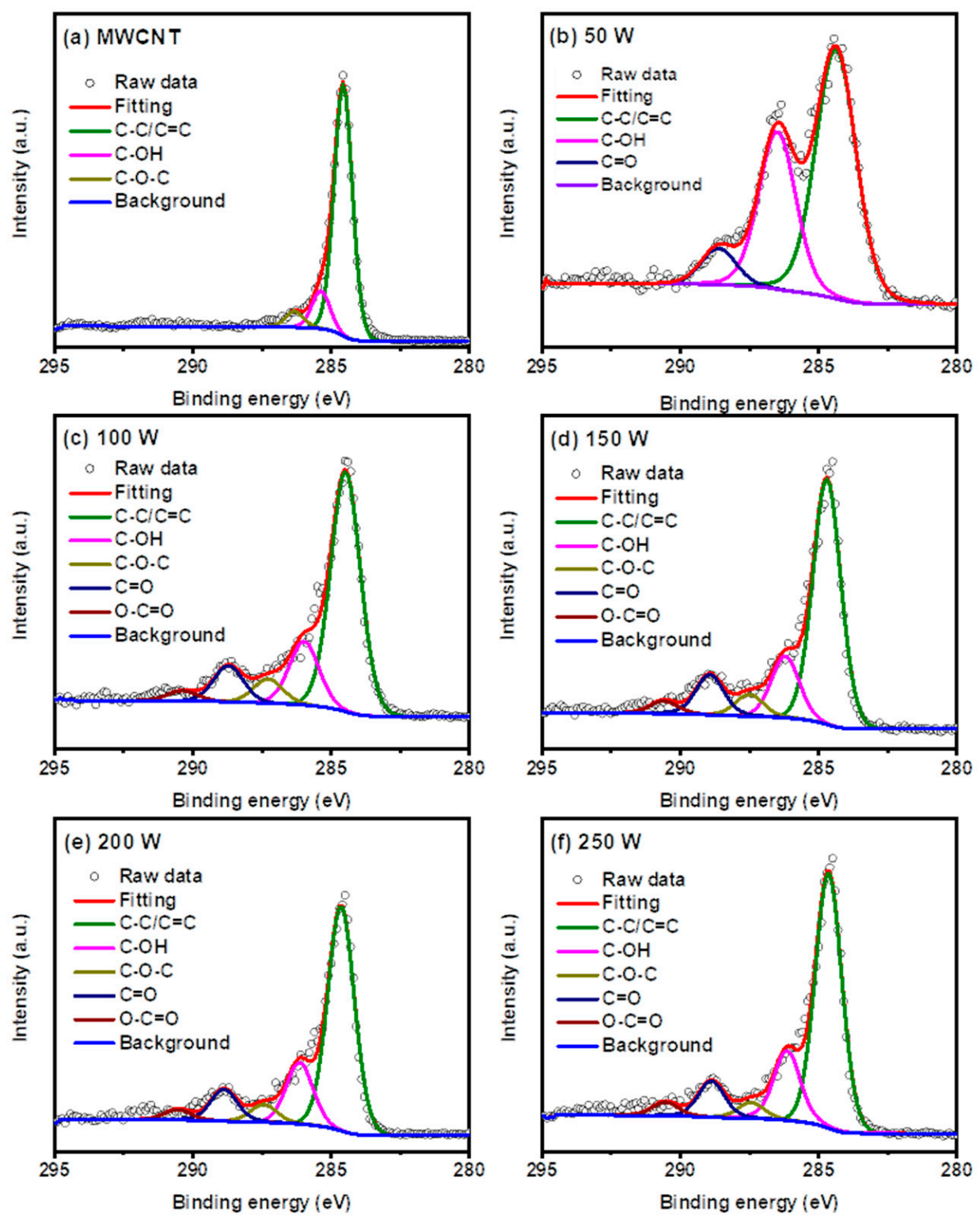

Figure 2. XPS spectra of (a) MWCNT, (b) GONR(50 W), (c) GONR(100 W), (d) GONR(150 W), (e) GONR(200 W), and (f) GONR(250 W) before and after the microwave-assisted unzipping process at different microwave powers.

\subsection{Electrochemical Measurements}

The cyclic voltammetry (CV) curves in Figure 3a-e show the dark currents and photocurrents of UA oxidation, using GONR(50-250 W) on SPCEs before and after AM 1.5 light irradiation. The electrolyte was $0.1 \mathrm{M}$ phosphate-buffered saline (PBS) containing $0.3 \mathrm{mM}$ $\mathrm{UA}$, and the scan rate remained at $50 \mathrm{mV} / \mathrm{s}$. The oxidation peaks of UA were located at $0.35 \mathrm{~V}$ to $0.39 \mathrm{~V}$. The increase in Faradaic currents was gradually raised from $34.1 \%$ $(\mathrm{GONR}(50 \mathrm{~W}))$ to $50.0 \%(\mathrm{GONR}(200 \mathrm{~W}))$, and then was dropped to $31.4 \%(\mathrm{GONR}(250 \mathrm{~W}))$. Among all catalysts, GONR(200 W) exhibited both the highest Faradaic dark current as well as the highest photocurrent. Its Faradaic dark current of $97.7 \mathrm{~mA}$ could grow up to $146.5 \mathrm{~mA}$ under light irradiation, demonstrating that GONR(200 W) was our best sample at this stage. In Figure 3f, using the low dark Faradaic current as the denominator for calculating the increase led to a more extensive error bar than the high dark Faradaic current. Conversely, we summarized the error bars in the increase from the experimental data by repeating the procedure five times. Consequently, they may appear to have had a wider distribution. The $\mathrm{CV}$ results for detecting ascorbic acid, dopamine, and UA are 
displayed in Figure S3 before and after light (AM 1.5) irradiation using GONR(200 W). Unlike UA oxidation, there was no obvious photo-enhanced phenomenon for ascorbic acid and dopamine detection. Therefore, in following experiments, we only focused on the UA oxidation reaction. It is worthwhile to mention that the double-layer CV curves with no UA added are recorded in Figure S4, indicating that GONR(200 W) could have also owned the largest capacitance among all ribbons.
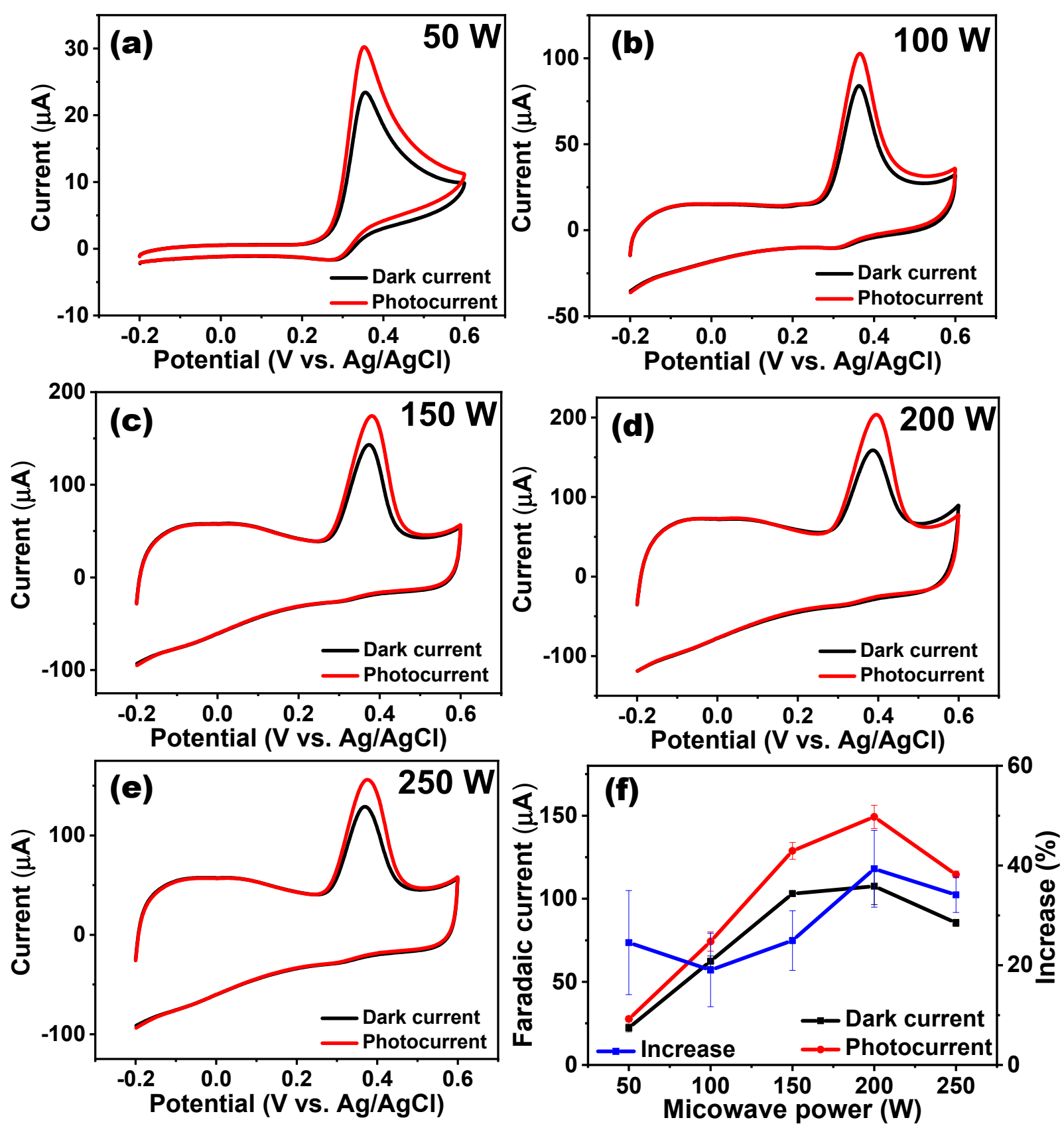

Figure 3. Cyclic voltammograms of (a) GONR(50 W), (b) GONR(100 W), (c) GONR(150 W), (d) GONR(200 W), and (e) GONR(250 W) with or without light (AM 1.5) illumination. (electrolyte: $0.3 \mathrm{mM} \mathrm{UA}+0.1 \mathrm{M}$ PBS, scan rate: $50 \mathrm{mV} / \mathrm{s}$ ) (f) The corresponding summary figure of Faradaic currents from Figure 3a to Figure 3e. The screen-printed carbon electrode was used for preparing the working electrode in Figure 3.

Figure 4a shows that the anodic oxidation peak current without light irradiation was $128.1 \mu \mathrm{A}$ at a potential of approximately $0.38 \mathrm{~V}$. After irradiation with blue light of $420 \mathrm{~nm}$ at an intensity of $5 \mathrm{~mW} / \mathrm{cm}^{2}$ through LED, the anodic current rose to $149.4 \mu \mathrm{A}$, and the 
Faradaic current was increased by approximately $22.7 \%$. Under subsequent blue light irradiations at different intensities of 10, 20,40,60, and $80 \mathrm{~mW} / \mathrm{cm}^{2}$, the Faradaic currents were increased by $28.7 \%, 35.7 \%, 41.4 \%, 45.1 \%$, and $46.9 \%$, respectively. Thus, the Faradaic current increased with the light intensity, but at a gradually decreasing rate. Conversely, Figure $4 \mathrm{~b}$ displays a CV plot under red light $(656 \mathrm{~nm})$ irradiation. At a potential of $0.38 \mathrm{~V}$, the anodic peak current without irradiation was $122.8 \mu \mathrm{A}$. After red light irradiation with an intensity of $5 \mathrm{~mW} / \mathrm{cm}^{2}$, the anodic current rose to $151.93 \mu \mathrm{A}$, and the Faradaic current increased by approximately $32.16 \%$. Similarly, the Faradaic currents were increased by $44.8 \%, 55.1 \%, 62.7 \%, 66.7 \%$, and $69.6 \%$ under the subsequent red light irradiation at the intensities of 10, 20, 40,60, and $80 \mathrm{~mW} / \mathrm{cm}^{2}$. Thus, under both blue and red light irradiation, the GONR $(200 \mathrm{~W})$ exhibited significant improvement in photo-assisted electrochemical biosensing. This phenomenon of using red light followed a similar trend observed under blue light irradiation, that is, the Faradaic current increased with the light intensity at a decreasing rate. In order to more carefully investigate this, six wavelengths from an LED were utilized for PEC experiments in addition to the original AM 1.5 light source. Figure 5a,c shows the processed Faradaic current data in $\mathrm{CV}$ measurement under light irradiations of $420,455,505,530,590$, and $656 \mathrm{~nm}$ at different intensities. For example, when choosing the wavelength of $420 \mathrm{~nm}$, we started the measurements by gradually increasing the light intensities from $5,10,20,40,60$ to $80 \mathrm{~mW} / \mathrm{cm}^{2}$. After finishing the $420 \mathrm{~nm}$, we moved to the following wavelengths of $455,505,530,590$, and $656 \mathrm{~nm}$, one by one. On the contrary, we started from $420 \mathrm{~nm}$ to $656 \mathrm{~nm}$ in Figure 5a, but reversed the sequences from $656 \mathrm{~nm}$ to 420 $\mathrm{nm}$ in Figure $5 \mathrm{c}$. Irrespective of the wavelengths of light, we clearly observed that the lightassisted Faradaic current gradually increased with the light intensity, but at a gradually decreasing rate under all six wavelengths. Notably, the increase in the Faradaic current decreased after changing wavelengths, presumably due to the reduced catalyst activity over the extended experimental time. Figure $5 b$,d shows that the increase in the photo-assisted Faradaic current could be independent of wavelengths. Therefore, this suggests that light intensity had a stronger effect than the wavelength on enhancing the photo-assisted Faradaic current. As seen in Figure 5, we always started the experiments from low light intensity to high intensity for one specific wavelength. The total experiment time for either Figure $5 \mathrm{a}, \mathrm{b}$ or Figure $5 \mathrm{c}, \mathrm{d}$ took approximately $7 \mathrm{~h}$. It was observed that the data from the low light intensity of $5 \mathrm{~mW} / \mathrm{cm}^{2}$ usually had a more expansive error bar. Finally, differential pulse voltammetry (DPV) results from when we changed UA concentrations with or without blue light $\left(80 \mathrm{~mW} / \mathrm{cm}^{2}\right)$ irradiation are presented in Figure S5. Under a dark circumstance, it was found that the anodic oxidation peak current was barely detectable at relatively low UA concentrations, that is, concentrations below $10 \mu \mathrm{M}$. The sensitivity displayed in Figure S5 is the slope divided by the carbon electrode's geometry area (3 $\mathrm{mm}$ diameter circle). The calculated sensitivity was $2.69 \mu \mathrm{A} / \mu \mathrm{Mcm}^{2}$ for the dark current. Figure S5c shows the response current depending on UA concentration under blue light $\left(80 \mathrm{~mW} / \mathrm{cm}^{2}\right)$ irradiation. The calculated sensitivity was $5.52 \mu \mathrm{A} / \mu \mathrm{Mcm}^{2}$ below $200 \mathrm{mM}$. It shows a considerably higher sensitivity to low-concentration UA with light irradiation compared to that without light irradiation. A summary on the average conversion efficiencies in cyclic voltammograms of GONR(200 W) with LED irradiation under different wavelengths and light intensities is depicted in Figure 6, with detailed information provided in Figure S6 and Tables S1-S4. There are two examples for calculating the conversion efficiencies displayed in Supplementary Tables S1 and S2. In Supplementary Table S1, we dealt with the case for $420 \mathrm{~nm}$ with $5 \mathrm{~mW} / \mathrm{cm}^{2}$. In Supplementary Table S2, we turned to $656 \mathrm{~nm}$ with $80 \mathrm{~mW} / \mathrm{cm}^{2}$. All the exact numbers can be found in the Supplementary Tables S3 and S4. On the one hand, the photon with the lower wavelength and higher energy could exhibit the better average conversion efficiency. On the other hand, the photon from the lower light intensity at the same wavelength could also own better conversion efficiency. Therefore, we can observe that the best average photon-to-electron conversion efficiency is over $20.00 \%$ for the short wavelength of $420 \mathrm{~nm}$ and the low intensity of $5 \mathrm{~mW} / \mathrm{cm}^{2}$. 

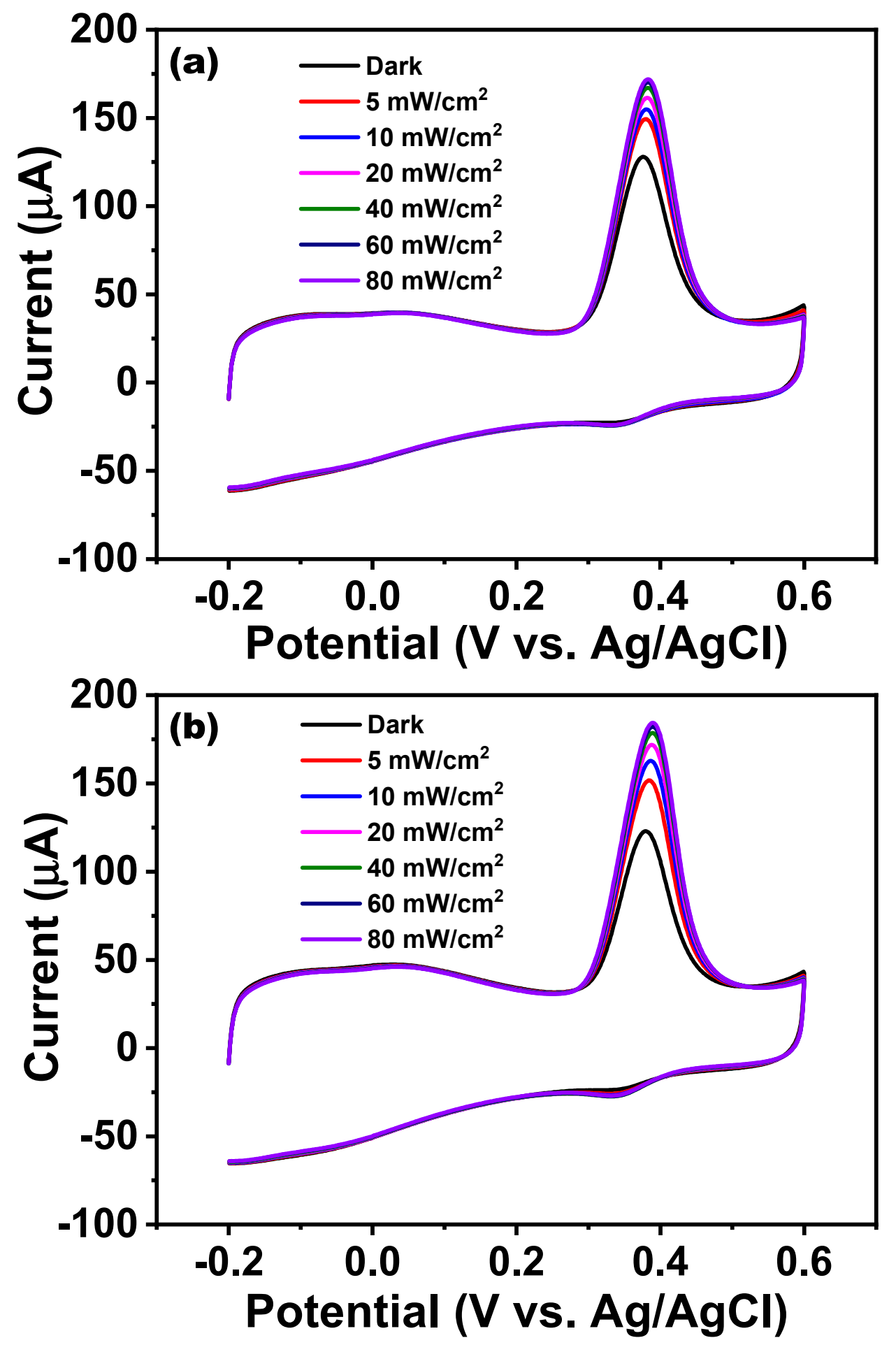

Figure 4. Cyclic voltammograms of $\operatorname{GONR}(200 \mathrm{~W})$ with or without light (LED) illumination using the wavelength of (a) $420 \mathrm{~nm}$ and (b) $656 \mathrm{~nm}$. (Electrolyte: $0.3 \mathrm{mM} \mathrm{UA}+0.1 \mathrm{M} \mathrm{PBS,} \mathrm{scan} \mathrm{rate:} 50 \mathrm{mV} / \mathrm{s}$ ). The light intensity of LED increases from $5 \mathrm{~mW} / \mathrm{cm}^{2}$ to $80 \mathrm{~mW} / \mathrm{cm}^{2}$. The glassy carbon electrode was used for preparing the working electrode in Figure 4. 

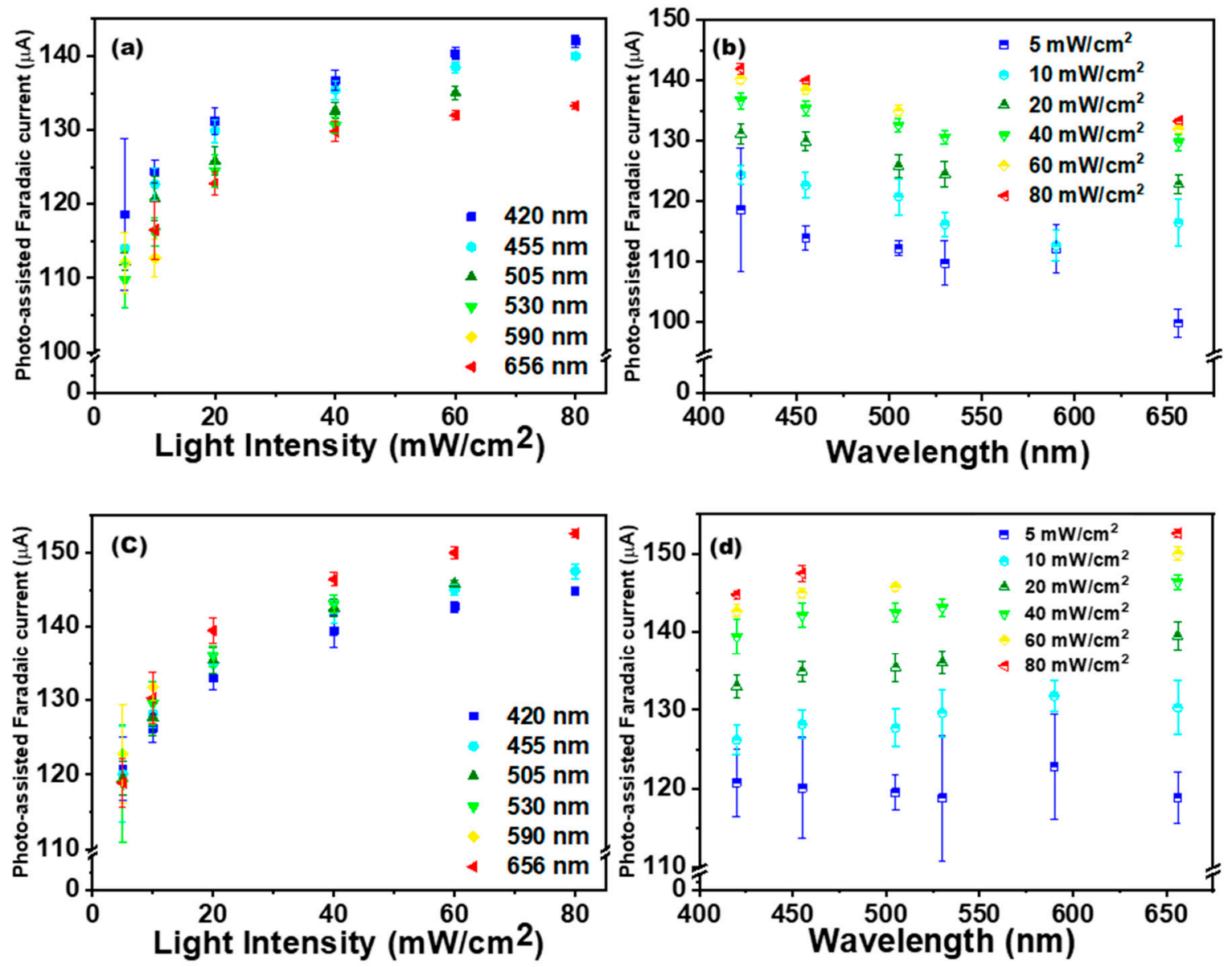

Figure 5. The summary figures of Faradaic currents in cyclic voltammograms of GONR(200 W) with light (LED) illumination using $(\mathbf{a}, \mathbf{c})$ different wavelengths and $(\mathbf{b}, \mathbf{c})$ light intensities. $(\mathbf{a}, \mathbf{b})$ The sequences of wavelength selections switch from $420 \mathrm{~nm}$ to $656 \mathrm{~nm}$. (c,d) The sequences switch from $656 \mathrm{~nm}$ to $420 \mathrm{~nm}$.

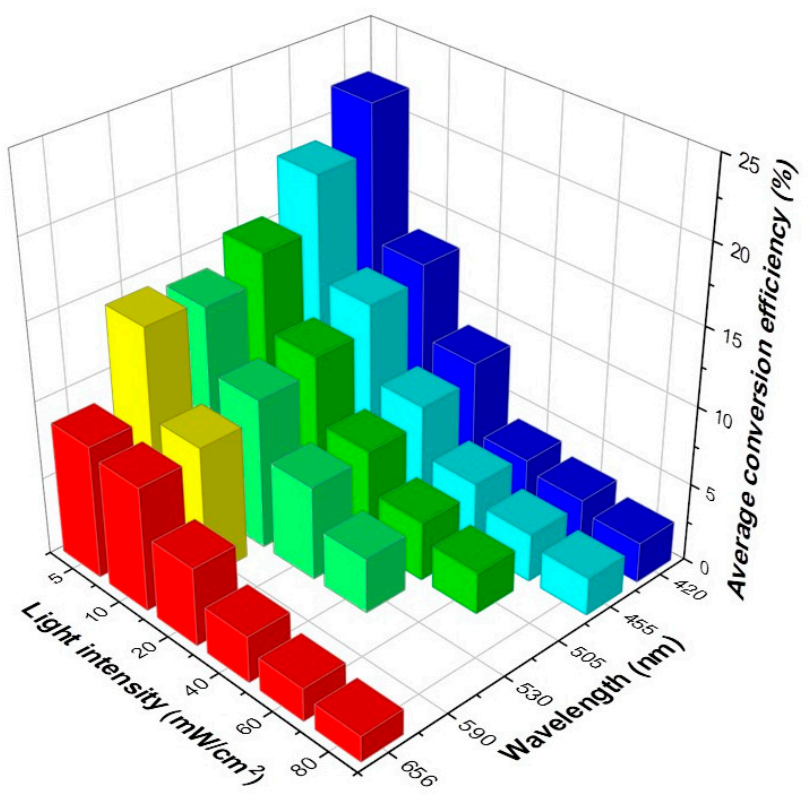

Figure 6. The summary figure of average conversion efficiencies in cyclic voltammograms of GONR(200 W) with light (LED) illumination using different wavelengths and light intensities derived from Figure 5. 


\section{Conclusions}

In summary, we have applied a microwave heating process to unzip MWCNTs into GONRs in a mixed acid solution. The GONRs were used to modify a working electrode for the photo-assisted biosensing of UA. The GONR $(200 \mathrm{~W})$ displayed better photo-enhanced electrochemical UA oxidation activity than other nanoribbons. Furthermore, irradiating the electrode surface with different light sources increased the response current over that obtained in a dark environment. For $0.3 \mathrm{mM}$ UA and a light intensity of $5 \mathrm{~mW} / \mathrm{cm}^{2}$, the Faradaic current of $\operatorname{GONR}(200 \mathrm{~W})$ increased by approximately $20.0 \%$, irrespective of light wavelengths. At a light intensity of $80 \mathrm{~mW} / \mathrm{cm}^{2}$, the Faradaic current was increased by around $40.0 \%$, suggesting that light intensity plays a significant role in our experiments. A DPV experiment showed an approximately two-fold increase in sensitivity from $2.687 \mu \mathrm{A} / \mu \mathrm{Mcm}^{2}$ to $5.517 \mu \mathrm{A} / \mu \mathrm{Mcm}^{2}$ under light irradiation at low concentrations. Therefore, it is believed that GONRs exhibited an excellent photocurrent response under light illumination for PEC biosensing applications.

Supplementary Materials: The following are available online at https:/ /www.mdpi.com/article/10 .3390/nano11102693/s1, Figure S1: Raman spectra of MWCNT and GONRs, Figure S2: XPS bonding ratios of MWCNT and GONRs. Figure S3: CVs of GONR(200 W)., Figure S4: CVs of GONRs. Figure S5: DPVs of GONR(200 W). Figure S6: Conversion efficiencies of GONR(200 W), Table S1: Conversion efficiency (\%) calculation for $420 \mathrm{~nm}$ with $5 \mathrm{~mW} / \mathrm{cm}^{2}$, Table S2: Conversion efficiency (\%) calculation for $656 \mathrm{~nm}$ with $80 \mathrm{~mW} / \mathrm{cm}^{2}$, Table S3: Calculation details when light illumination was carried out from $420 \mathrm{~nm}$ to $656 \mathrm{~nm}$, Table S4: Calculation details when light illumination was carried out from $656 \mathrm{~nm}$ to $420 \mathrm{~nm}$.

Author Contributions: Conceptualization, C.-L.S. and Y.-J.L.; methodology, C.-H.L. and C.-H.K.; formal analysis, C.-L.S., T.-C.C., C.-Y.C. and Y.-J.L.; investigation, C.-H.L., C.-H.K., C.-W.H. and D.D.N.; resources, C.-L.S. and Y.-J.L.; writing-original draft preparation, C.-L.S., T.-C.C., C.-Y.C. and Y.-J.L.; writing-review and editing, C.-L.S. and Y.-J.L.; supervision, C.-L.S.; project administration, C.-L.S.; funding acquisition, C.-L.S. and Y.-J.L. All authors have read and agreed to the published version of the manuscript.

Funding: This research was funded by Chang Gung Memorial Hospital, grant number BMRPA64 and grand number CMRPD2H0321.

Acknowledgments: This work was supported by the grants from the Ministry of Science and Technology and Chang Gung Memorial Hospital (BMRPA64 and CMRPD2H0321).

Conflicts of Interest: The authors declare no conflict of interest.

\section{References}

1. Rashid, M.H.; Koel, A.; Rang, T. Simulations of Graphene Nanoribbon Field Effect Transistor for the Detection of Propane and Butane Gases: A First Principles Study. Nanomaterials 2020, 10, 98. [CrossRef] [PubMed]

2. Damasceno, D.A.; Rajapakse, R.K.N.D.N.; Mesquita, E. Atomistic Modelling of Size-Dependent Mechanical Properties and Fracture of Pristine and Defective Cove-Edged Graphene Nanoribbons. Nanomaterials 2020, 10, 1422. [CrossRef]

3. Lin, S.-Y.; Tran, N.T.T.; Lin, M.-F. Diversified Phenomena in Metal- and Transition-Metal-Adsorbed Graphene Nanoribbons. Nanomaterials 2021, 11, 630. [CrossRef] [PubMed]

4. Li, P.; Liu, J.; Her, S.; Zal Nezhad, E.; Lim, S.; Bae, S. Synthesis of Highly-Dispersed Graphene Oxide Nanoribbons-Functionalized Carbon Nanotubes-Graphene Oxide (GNFG) Complex and Its Application in Enhancing the Mechanical Properties of Cementitious Composites. Nanomaterials 2021, 11, 1669. [CrossRef]

5. Suzuki, K.; Nakagawa, R.; Zhang, Q.; Miura, H. Development of Highly Sensitive Strain Sensor Using Area-Arrayed Graphene Nanoribbons. Nanomaterials 2021, 11, 1701. [CrossRef] [PubMed]

6. Sivula, K.; van de Krol, R. Semiconducting materials for photoelectrochemical energy conversion. Nat. Rev. Mater. 2016, 1, 15010. [CrossRef]

7. Yu, H.; Shi, R.; Zhao, Y.; Waterhouse, G.I.; Wu, L.Z.; Tung, C.H.; Zhang, T. Smart Utilization of Carbon Dots in Semiconductor Photocatalysis. Adv. Mater. 2016, 28, 9454-9477. [CrossRef]

8. Roger, I.; Shipman, M.A.; Symes, M.D. Earth-abundant catalysts for electrochemical and photoelectrochemical water splitting. Nat. Rev. Chem. 2017, 1, 0003. [CrossRef]

9. Li, X.; Zhu, L.; Zhou, Y.; Yin, H.; Ai, S. Enhanced Photoelectrochemical Method for Sensitive Detection of Protein Kinase A Activity Using $\mathrm{TiO}_{2} / \mathrm{g}-\mathrm{C}_{3} \mathrm{~N}_{4}$, PAMAM Dendrimer, and Alkaline Phosphatase. Anal. Chem. 2017, 89, 2369-2376. [CrossRef] 
10. Lin, Y.; Zhou, Q.; Tang, D.; Niessner, R.; Knopp, D. Signal-On Photoelectrochemical Immunoassay for Aflatoxin B1 Based on Enzymatic Product-Etching $\mathrm{MnO}_{2}$ Nanosheets for Dissociation of Carbon Dots. Anal. Chem. 2017, 89, 5637-5645. [CrossRef]

11. Shu, J.; Qiu, Z.; Lv, S.; Zhang, K.; Tang, D. Plasmonic Enhancement Coupling with Defect-Engineered TiO $\mathrm{T}_{2}-\mathrm{x}$ : A Mode for Sensitive Photoelectrochemical Biosensing. Anal. Chem. 2018, 90, 2425-2429. [CrossRef]

12. Zhou, Q.; Lin, Y.; Zhang, K.; Li, M.; Tang, D. Reduced graphene oxide/ $\mathrm{BiFeO}_{3}$ nanohybrids-based signal-on photoelectrochemical sensing system for prostate-specific antigen detection coupling with magnetic microfluidic device. Biosens. Bioelectron. 2018, 101, 146-152. [CrossRef]

13. Sun, M.; Zhu, Y.; Yan, K.; Zhang, J. Dual-mode visible light-induced aptasensing platforms for bleomycin detection based on $\mathrm{CdS}-\mathrm{In}_{2} \mathrm{~S}_{3}$ heterojunction. Biosens. Bioelectron. 2019, 145, 111712. [CrossRef]

14. Qian, Y.; Feng, J.; Wang, H.; Fan, D.; Jiang, N.; Wei, Q.; Ju, H. Sandwich-type signal-off photoelectrochemical immunosensor based on dual suppression effect of $\mathrm{PbS}$ quantum dots $/ \mathrm{Co}_{3} \mathrm{O}_{4}$ polyhedron as signal amplification for procalcitonin detection. Sens. Actuators B Chem. 2019, 300, 127001. [CrossRef]

15. Zhu, X.; Gao, L.; Tang, L.; Peng, B.; Huang, H.; Wang, J.; Yu, J.; Ouyang, X.; Tan, J. Ultrathin PtNi nanozyme based self-powered photoelectrochemical aptasensor for ultrasensitive chloramphenicol detection. Biosens. Bioelectron. 2019, 146, 111756. [CrossRef]

16. Deng, K.; Wang, H.; Xiao, J.; Li, C.; Zhang, S.; Huang, H. Polydopamine nanospheres loaded with l-cysteine-coated cadmium sulfide quantum dots as photoelectrochemical signal amplifier for PSA detection. Anal. Chim. Acta 2019, 1090, 143-150. [CrossRef]

17. Zhu, Y.; Xu, Z.; Gao, J.; Ji, W.; Zhang, J. An antibody-aptamer sandwich cathodic photoelectrochemical biosensor for the detection of progesterone. Biosens. Bioelectron. 2020, 160, 112210. [CrossRef] [PubMed]

18. Liu, Q.; Kim, J.; Cui, T. A highly sensitive photoelectrochemical sensor with polarity-switchable photocurrent for detection of trace hexavalent chromium. Sens. Actuators B Chem. 2020, 317, 128181. [CrossRef]

19. Zhang, Z.; Zhang, M.; Xu, Y.; Wen, Z.; Ding, C.; Guo, Y.; Hao, N.; Wang, K. Bi ${ }^{3+}$ engineered black anatase titania coupled with graphene for effective tobramycin photoelectrochemical detection. Sens. Actuators B Chem. 2020, 321, 128464. [CrossRef]

20. Ma, S.; Yang, Q.; Zhang, W.; Xiao, G.; Wang, M.; Cheng, L.; Zhou, X.; Zhao, M.; Ji, J.; Zhang, J.; et al. Silver nanoclusters and carbon dots based light-addressable sensors for multichannel detections of dopamine and glutathione and its applications in probing of parkinson's diseases. Talanta 2020, 219, 121290. [CrossRef] [PubMed]

21. Kawai, S.; Benassi, A.; Gnecco, E.; Söde, H.; Pawlak, R.; Feng, X.; Müllen, K.; Passerone, D.; Pignedoli, C.A.; Ruffieux, P.; et al. Superlubricity of graphene nanoribbons on gold surfaces. Science 2016, 351, 957-961. [CrossRef]

22. Ruffieux, P.; Wang, S.; Yang, B.; Sanchez-Sanchez, C.; Liu, J.; Dienel, T.; Talirz, L.; Shinde, P.; Pignedoli, C.A.; Passerone, D.; et al. On-surface synthesis of graphene nanoribbons with zigzag edge topology. Nature 2016, 531, 489-492. [CrossRef]

23. Yu, X.; Wang, B.; Gong, D.; Xu, Z.; Lu, B. Graphene Nanoribbons on Highly Porous 3D Graphene for High-Capacity and Ultrastable Al-Ion Batteries. Adv. Mater. 2017, 29, 1604118. [CrossRef]

24. Rizzo, D.J.; Veber, G.; Cao, T.; Bronner, C.; Chen, T.; Zhao, F.; Rodriguez, H.; Louie, S.G.; Crommie, M.F.; Fischer, F.R. Topological band engineering of graphene nanoribbons. Nature 2018, 560, 204-208. [CrossRef] [PubMed]

25. Vukojevic, V.; Djurdjic, S.; Ognjanovic, M.; Antic, B.; Kalcher, K.; Mutic, J.; Stankovic, D.M. RuO 2 /graphene nanoribbon composite supported on screen printed electrode with enhanced electrocatalytic performances toward ethanol and NADH biosensing Biosens. Bioelectron. 2018, 117, 392-397. [CrossRef]

26. Tang, Z.; He, J.; Chen, J.; Niu, Y.; Zhao, Y.; Zhang, Y.; Yu, C. A sensitive sandwich-type immunosensor for the detection of galectin-3 based on N-GNRs-Fe-MOFs@AuNPs nanocomposites and a novel AuPt-methylene blue nanorod. Biosens. Bioelectron. 2018, 101, 253-259. [CrossRef] [PubMed]

27. Li, Y.; He, J.; Chen, J.; Niu, Y.; Zhao, Y.; Zhang, Y.; Yu, C. A dual-type responsive electrochemical immunosensor for quantitative detection of PCSK9 based on n-C60-PdPt/N-GNRs and Pt-poly(methylene blue) nanocomposites. Biosens. Bioelectron. 2018, 101, 7-13. [CrossRef]

28. Feng, Q.; Zhao, X.; Guo, Y.; Liu, M.; Wang, P. Stochastic DNA walker for electrochemical biosensing sensitized with gold nanocages@graphene nanoribbons. Biosens. Bioelectron. 2018, 108, 97-102. [CrossRef]

29. Liu, Q.; Bao, J.; Yang, M.; Wang, X.; Lan, S.; Hou, C.; Wang, Y.; Fa, H. A core-shell MWCNT@rGONR heterostructure modified glassy carbon electrode for ultrasensitive electrochemical detection of glutathione. Sens. Actuators B Chem. 2018, 274, 433-440. [CrossRef]

30. Pajooheshpour, N.; Rezaei, M.; Hajian, A.; Afkhami, A.; Sillanpää, M.; Arduini, F.; Bagheri, H. Protein templated Au-Pt nanoclusters-graphene nanoribbons as a high performance sensing layer for the electrochemical determination of diazinon. Sens. Actuators B Chem. 2018, 275, 180-189. [CrossRef]

31. Sun, C.-L.; Chang, C.-T.; Lee, H.-H.; Zhou, J.; Wang, J.; Sham, T.-K.; Pong, W.-F. Microwave-Assisted Synthesis of a Core-Shell MWCNT-GONR Heterostructure for the Electrochemical Detection of Ascorbic Acid, Dopamine, and Uric Acid. ACS Nano 2011, 5, 7788-7795. [CrossRef]

32. Sun, C.L.; Su, C.H.; Wu, J.J. Synthesis of short graphene oxide nanoribbons for improved biomarker detection of Parkinson's disease. Biosens. Bioelectron. 2015, 67,327-333. [CrossRef]

33. Lan, Y.-W.; Torres, C.M., Jr.; Zhu, X.; Sun, C.-L.; Zhu, S.; Chen, C.-D.; Wang, K.-L. Self-aligned graphene oxide nanoribbon stack with gradient bandgap for visible-light photodetection. Nano Energy 2016, 27, 114-120. [CrossRef] 
34. Lin, T.E.; Lu, Y.J.; Sun, C.L.; Pick, H.; Chen, J.P.; Lesch, A.; Girault, H.H. Soft Electrochemical Probes for Mapping the Distribution of Biomarkers and Injected Nanomaterials in Animal and Human Tissues. Angew. Chem. Int. Ed. Engl. 2017, 56, 16498-16502. [CrossRef] [PubMed]

35. Zhang, R.; Zhang, C.; Zheng, F.; Li, X.; Sun, C.-L.; Chen, W. Nitrogen and sulfur co-doped graphene nanoribbons: A novel metalfree catalyst for high performance electrochemical detection of 2,4,6-trinitrotoluene (TNT). Carbon 2018, 126, 328-337. [CrossRef]

36. Liu, Y.-C.; Hung, Y.-H.; Liu, S.-F.; Guo, C.-H.; Liu, T.-Y.; Sun, C.-L.; Chen, H.-Y. Core-shell structured multiwall carbon nanotubegraphene oxide nanoribbon and its N-doped variant as anodes for high-power microbial fuel cells. Sustain. Energy Fuels 2020, 4, 5339-5351. [CrossRef]

37. Wang, T.; Wang, Z.; Salvatierra, R.V.; McHugh, E.; Tour, J.M. Top-down synthesis of graphene nanoribbons using different sources of carbon nanotubes. Carbon 2020, 158, 615-623. [CrossRef]

38. Silver, A.; Kitadai, H.; Liu, H.; Granzier-Nakajima, T.; Terrones, M.; Ling, X.; Huang, S. Chemical and Bio Sensing Using Graphene-Enhanced Raman Spectroscopy. Nanomaterials 2019, 9, 516. [CrossRef]

39. Jakubowski, W.; Atraszkiewicz, R.; Nowak, D.; Batory, D.; Szymański, W.; Sobczyk-Guzenda, A.; Kaczmarek, Ł.; Kula, P.; Cłapa, M.; Warga, T.; et al. Optimization of Glutathione Adhesion Process to Modified Graphene Surfaces. Nanomaterials 2021, 11, 756. [CrossRef] 